\title{
Measurements of the Optical Constants of Scandium in the 50-1300eV Range
}

\author{
A. L. Aquila, F. Salmassi, E. M. Gullikson \\ Center for X-Ray Optics, Lawrence Berkeley National Laboratory, Berkeley, CA 94720 \\ F. Eriksson, J. Birch \\ Department of Physics, Linköping University, 58183 Linköping, Sweden
}

\begin{abstract}
Scandium containing multilayers have been produced with very high reflectivity in the soft x-ray spectrum. Accurate optical constants are required in order to model the multilayer reflectivity. Since there are relatively few measurements of the optical constants of Scandium in the soft $\mathrm{x}$-ray region we have performed measurements over the energy range of 50-1,300 eV. Thin films of Scandium were deposited by ion-assisted magnetron sputtering at Linkoping University and DC Magnetron sputtering at CXRO. Transmission measurements were performed at the Advanced Light Source beamline 6.3.2. The absorption coefficient was deduced from the measurements and the dispersive part of the index of refraction was obtained using the Kramers-Kronig relation. The measured optical constants are used to model the nearnormal incidence reflectivity of $\mathrm{Cr} / \mathrm{Sc}$ multilayers near the $\mathrm{Sc}_{2,3}$ edge.
\end{abstract}

Keywords: Index of refraction, EUV, soft x-ray, Sc

\section{INTRODUCTION}

Scandium is an important optical material in the extreme ultraviolet (EUV) and soft x-ray bands of the spectrum. In the EUV range of $25-35 \mathrm{eV}, \mathrm{Sc} / \mathrm{Si}$ multilayers have been fabricated with a near-normal incidence reflectivity above $50 \%$. In the soft x-ray region near $400 \mathrm{eV}$, the highest near-normal incidence reflectivity has been achieved ${ }^{1}$ with $\mathrm{Cr} / \mathrm{Sc}$ multilayer mirrors just below the $\mathrm{Sc}_{2,3}$ edges $(403.6 \mathrm{eV}, 398.7 \mathrm{eV})$. This energy is in the important "water window" between the Carbon K edge $(248.2 \mathrm{eV})$ and the Oxygen K edge $(543.1 \mathrm{eV})$ where proteins are absorbent and the watery cytoplasm is transparent.

Modeling the properties of multilayer mirrors requires an accurate knowledge of the material optical constants. In the case of Scandium there have been very few measurements in the soft x-ray region. The available tabulated data ${ }^{2}$ for Scandium published by Henke et al. in 1993 are based on theoretical calculations and interpolation from measurements on neighboring elements. These tables also do not include the fine structure near absorption edges. A comprehensive study of Scandium in the EUV and soft x-ray region would allow for better modeling of scandium multilayers.

This research tabulates the optical constants $\delta$ and $\beta$ for Scandium using freestanding thin film Scandium samples sandwiched between Silicon capping layers. Sample deposition and preparation is described in experiment subsection of this paper. The optical constant $\beta$ is determined from the absorption measurements of freestanding Scandium films. The measurements were carried out in a photon energy range of $50 \mathrm{eV}$ to $1,300 \mathrm{eV}$ using the Advanced Light Sources beamline 6.3.2 as described in the experimental section of this paper. From $\beta$ the Kramers-Kronig analysis was preformed to determine the real part of the index of refraction, $1-\delta$. Finally the determined optical constants are used to model the measured reflectance from a $\mathrm{Cr} / \mathrm{Sc}$ multilayer at energies which span the $\mathrm{Sc} \mathrm{L}$ edge. 


\section{EXPERIMENT}

\section{Sample Preparation}

The samples used in this experiment were Scandium films with thickness ranging from $50 \mathrm{~nm}$ to $500 \mathrm{~nm}$. The first samples were deposited at Linköping University on silicon nitride membranes. From the results of those measurements it was decided to fabricate freestanding films because of the close proximity of the $\mathrm{N} \mathrm{K}$ edge $(409.9 \mathrm{eV})$ to the Scandium Sc $\mathrm{L}_{2,3}$ edge $(403.6 \mathrm{eV}, 398.7 \mathrm{eV}$ ). The freestanding films were fabricated by depositing a trilayer of $\mathrm{Si} / \mathrm{Sc} / \mathrm{Si}$ on photoresist-coated wafers. After gluing a frame to the film the photoresist was dissolved by soaking in acetone. The freestanding films were made in Berkeley by DC Magnetron sputtering in a system with a base pressure of $10^{-7}$ Torr. The first samples fabricated in this way disintegrated in the acetone due to the high stress of the films. In order to minimize the stress, the Argon pressure was varied in the range of 2.3 to 4.5 mTorr depending on the thickness. An oxidation barrier of $5 \mathrm{~nm}$ of Silicon was deposited immediately before and after the Sc with out breaking vacuum. Pieces of the silicon wafers that were not used for filters were used in Rutherford Back Scattering (RBS) measurements and in soft x-ray reflectance measurements to determine the thickness of the Sc layer, and contaminants in the film. The film thickness was determined to be: $49 \mathrm{~nm}, 100 \mathrm{~nm}$, and $192 \mathrm{~nm}$ to an accuracy of $5 \%$ using soft x-ray reflectivity versus angle. However the $486 \mathrm{~nm}$ sample was too thick for this method and RBS measurements were used to determine its thickness. The results of the RBS measurement showed that scandium samples were $99.9 \%$ pure Scandium which was consistent with the target manufacturers purity specification. There were no detectable amounts of Argon or Oxygen in the samples within the limits of $<1.5 \%$ and $<1.0 \%$ respectively.

\section{Instrumentation}

The transmission measurements were preformed using the Calibrations and Standards beamline ${ }^{3}$ at the Advanced Light Source, beamline 6.3.2. This is one of the synchrotron's bending magnet beamlines, which operates in the range of 50 $\mathrm{eV}$ to $1,300 \mathrm{eV}$ with a resolving power up to 7000 . The high spectral purity of this beamline was essential to making the accurate transmission measurements required in this work. Second order and stay light is suppressed using various filters and at low energies $(<600 \mathrm{eV})$ higher order light (third and fourth orders) is suppressed using a grazing incident triple reflection order suppressor. The end result is a beam with a spectral purity greater than $99 \%$. The photon energy near the Sc L edge was calibrated using the absorption spectrum of nitrogen gas ${ }^{4}$ and in this range is accurate to 0.02 $\mathrm{eV}$.

\section{RESULTS}

Transmission measurements of the four $\mathrm{Si} / \mathrm{Sc} / \mathrm{Si}$ films were preformed from $50 \mathrm{eV}$ to $1,300 \mathrm{eV}$ with a variable spacing of points depending on absorption features of the samples. For example around $400 \mathrm{eV}$ near the Scandium edge a spacing of $.2 \mathrm{eV}$ was used while at $1,200 \mathrm{eV}$ a spacing of $5 \mathrm{eV}$ was used. Extra care for absolute accuracy and high resolution was given to measurements near the Scandium edge to resolve the fine structure. Measurement reproducibility was $.1 \%$ for measurements greater than $2 \%$ transmission. The transmission measurements were obtained by the expression:

$$
\mathrm{T}=((\mathrm{I}-\mathrm{D}) / \mathrm{R}) /\left(\left(\mathrm{I}_{0}-\mathrm{D}_{0}\right) / \mathrm{R}_{0}\right)
$$

Where I is the photodiode current (proportional to the transmission through the film), D is the average value of the dark current (the current noise when no light is in the chamber), and $\mathrm{R}$ is the ring current. We double normalize using ring current because of its proportional decay with the number of photons. The $\mathrm{I}_{0}, \mathrm{D}_{0}, \mathrm{R}_{0}$ are the same as the incident (transmission) data except that the transmission measurement is taken when an empty filter holder. $\mathrm{D}$ and $\mathrm{D}_{0}$ may be different values depending on the amplification gain needed for I and $\mathrm{I}_{0}$.

Once the transmission measurements were obtained the absorption coefficient $\mu$ in units $\mathrm{of}\left[\mathrm{cm}^{2} / \mathrm{g}\right]$ was obtained through the equation:

$$
\mathrm{T}=\mathrm{T}_{0} \exp (-\mu \rho x)
$$




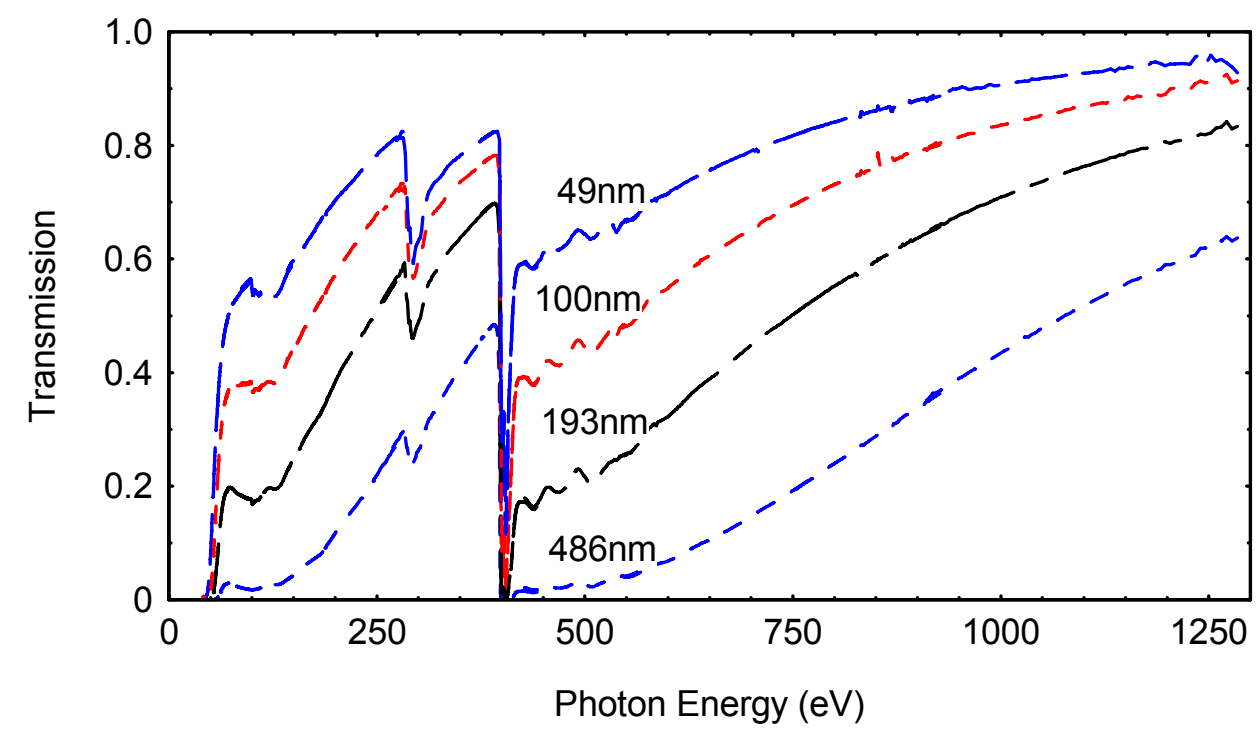

Figure 1. The transmission versus energy of four $\mathrm{Si} / \mathrm{Sc} / \mathrm{Si}$ films with $\mathrm{Sc}$ thickness of $49,100,193$, and $486 \mathrm{~nm}$. The structure is due to the L-absorption edges of Silicon $(100 \mathrm{eV})$ and $\mathrm{Sc}(400 \mathrm{eV})$ and the K edge of carbon at $284 \mathrm{eV}$.

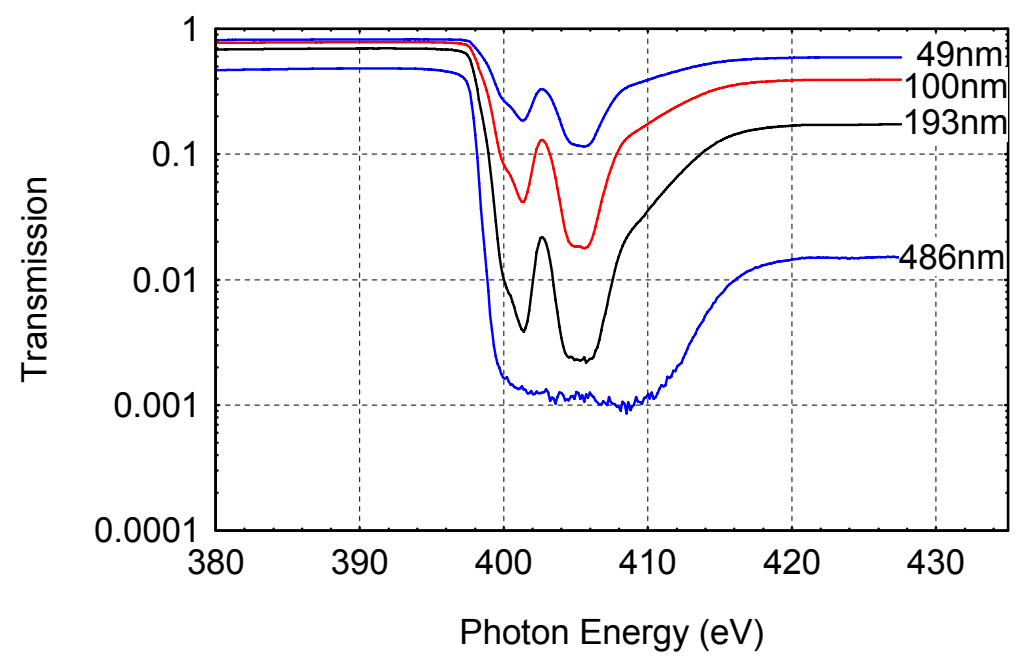

Figure 2. The transmission near the Scandium $\mathrm{L}_{2,3}$ edge. The two dips in reflectivity represent the two edges. Also note that on the thick $486 \mathrm{~nm}$ sample the two edges are washed out due to the background of scattered light. 


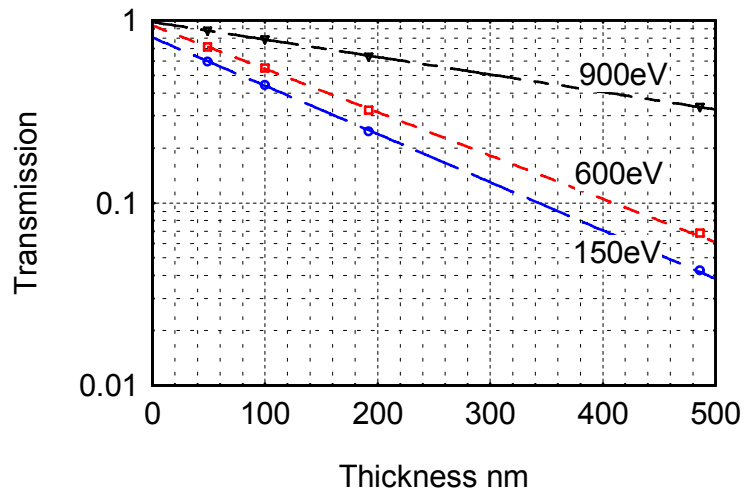

Figure 3. Fitting of $\mu$ versus thickness at photon energies of 150,600 , and $900 \mathrm{eV}$. The dashed lines are fits where the slope is $-\mu \rho$.

Where $\mathrm{x}$ is the thickness $[\mathrm{cm}]$ and $\rho$ is the density of Sc. We assumed the films had the bulk density of $2.99 \mathrm{~g} / \mathrm{cm}^{3}$. This was checked by comparing the thickness obtained by x-ray reflectivity and RBS for the $100 \mathrm{~nm}$ film. Plotting $\mathrm{ln}(\mathrm{T})$ verses sample thickness for each photon energy gives a linear relationship where the slope is $-\mu \rho$ and the intercept is $\mathrm{T}_{0}$. Examples of determining $\mu$ at three different energies are shown in figure 3 . The linear fits of the data were very good and yielded standard deviations around 0.005 . When the transmission fell below $1 \%$ the measurement was not used in the linear fit analysis due to the significant influence of scattered and higher order light.

$\mathrm{T}_{0}$ is the extrapolation to zero film thickness and ideally this would be unity. However $\mathrm{T}_{0}$ is less than one because of the silicon capping layer and oxide and the residual photoresist. Plotting $\mathrm{T}_{0}$ verses photon energy thus provides an indication of the contamination of the samples (figure 4). It also assumes that the thickness of the capping layer and photoresist are the same for all samples. Because of the method of deposition of the thin foil this assumption is justified. The fit of the contamination of the samples yields $20 \mathrm{~nm}$ of residual photoresist and the $10 \mathrm{~nm}$ of $\mathrm{Si} / \mathrm{SiO} \mathrm{O}_{2}$ capping layer. There is some distortion around the Scandium L edge caused by slight errors in sample thickness. Small discontinuities occurred when the transmission of a sample dropped below $1 \%$ and the data was no longer used. These discontinuities were more apparent in the $\mathrm{T}_{0}$ than in $\mu$.

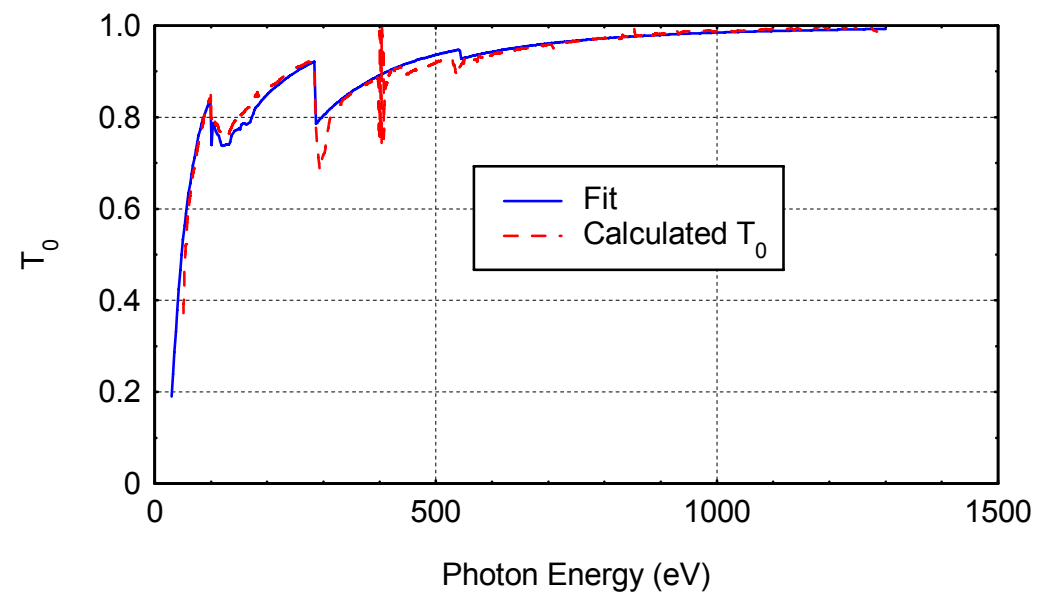

Figure 4. The transmission extrapolated to zero Sc thickness, $\mathrm{T}_{0}$ as a function of photon energy. Please note the Silicon $\mathrm{L}_{2,3}$ edge $(100 \mathrm{eV})$, the carbon $\mathrm{K}$ edge $(284 \mathrm{eV})$ and the oxygen $\mathrm{K}$ edge $(543 \mathrm{eV})$. The lack of an Argon $\mathrm{L}_{2,3}$ edge $(250 \mathrm{eV}, 248 \mathrm{eV})$ is consistent

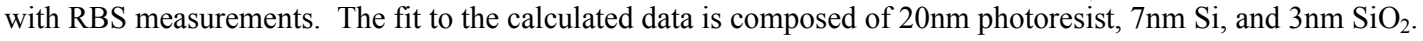


Looking at $\mu$ as a function of photon energy (figure 5) there are some striking differences from the previous tabulated measurements. There is a large absorption peak near the edge that is a factor of 4 larger than the tabulated data from the CXRO webpage ${ }^{5}$. The strong absorption near edge structure is explained by the fact that Scandium has an almost empty $3 \mathrm{~d}$ band and thus a large density of empty $\mathrm{d}$ states for $\mathrm{L}$ edge transitions from the $2 \mathrm{p}$ shell.

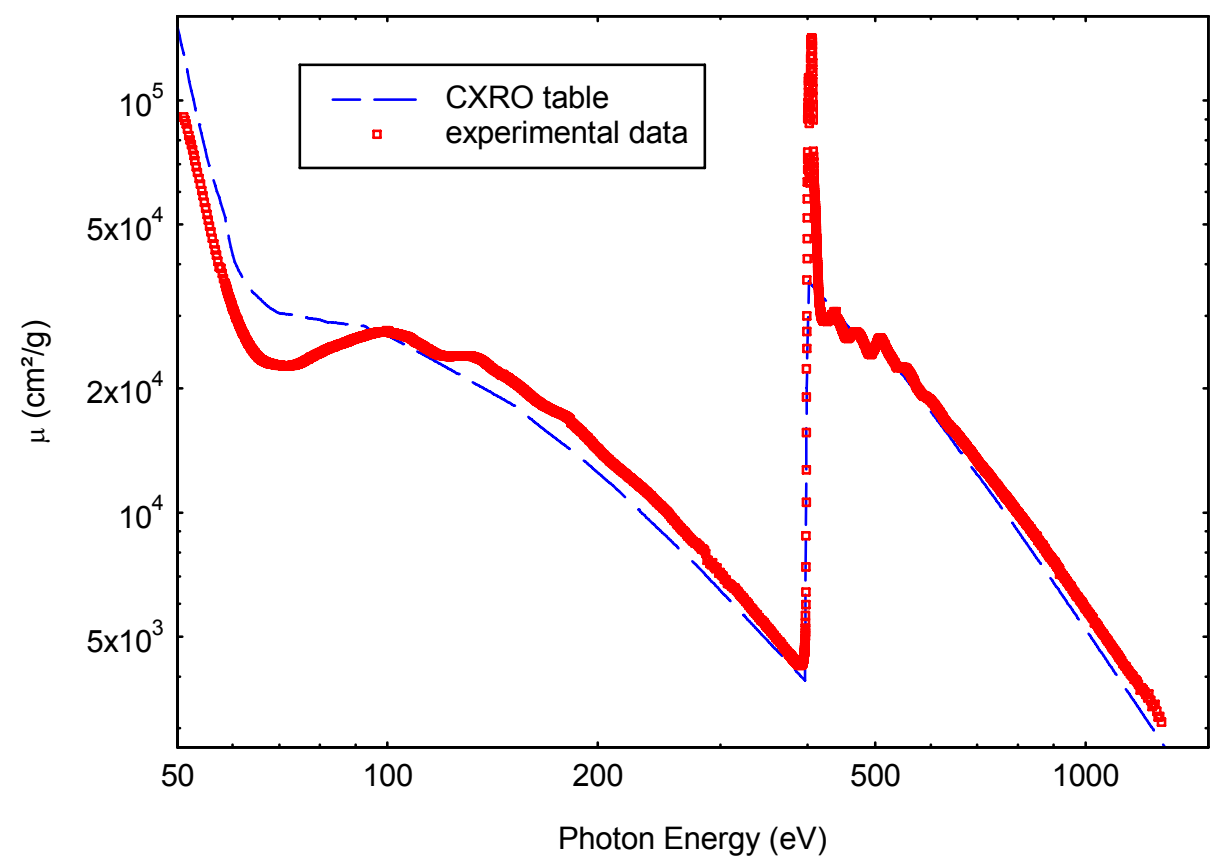

Figure 5. This measured absorption coefficient (points) compared with the tabulated values of scandium absorption from reference 2. Notice the strong near edge effect at the Scandium L edge that was not incorporated in the tabulated values.

From $\mu$ it is straightforward to calculate the complex part of the index of refraction, $\beta$ where $n=1-\delta+i \beta$ by the formula:

$$
\beta=\lambda \mu / 4 \pi
$$

The real part was calculated using Kramers-Kronig analysis:

$$
\delta=(2 / \pi) \int_{0}^{\infty} \frac{E^{\prime} \beta\left(E^{\prime}\right)}{E^{2}-E^{\prime 2}} d E^{\prime}
$$

To perform the above integral, the absorption spectrum, $\beta$, of Scandium was needed over a wide range of energy. The values calculated in this work were combined with the tables from the CXRO web page at energies above $1,300 \mathrm{eV}$ and the measurements of Uspenski et. $a l^{6}$ below $50 \mathrm{eV}$ and the values given by Weaver ${ }^{7}$ for energies from $0.1 \mathrm{eV}$ to $5 \mathrm{eV}$. As a self-consistency check of the data the sum rule was calculated by integrating $\mu$ as described in previous works ${ }^{8}$. The integral gave a value of 20.35 which is only $3 \%$ lower than the atomic number of Sc, $Z=21$.

The derived optical constants are compared the tabulated values in Fig. 6 . The values of both $\beta$ and $\delta$ are in good agreement with recent measurements by Uspenski et. al. The optical constants in the region near the Sc L edge are 
shown in Fig. 7. The strong absorption peak in $\beta$ causes $\delta$ to go more negative for energies just below the edge. This would tend to increase the theoretical reflectance of Sc containing multilayer mirrors in this region.

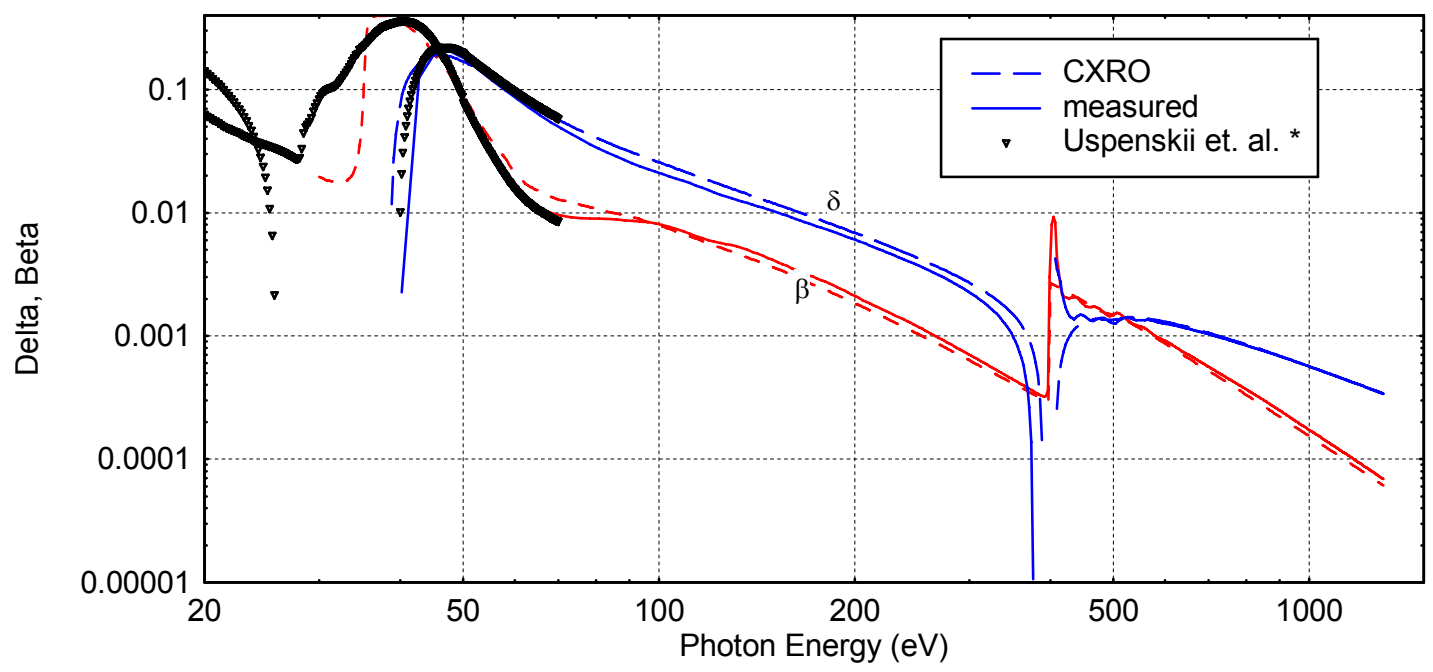

Figure 6. This graph shows the optical constants for $\delta$ and $\beta$ over the entire EUV and soft X-ray range. Notice the good agreement with the results of Uspenskii et. al. for lower photon energies.

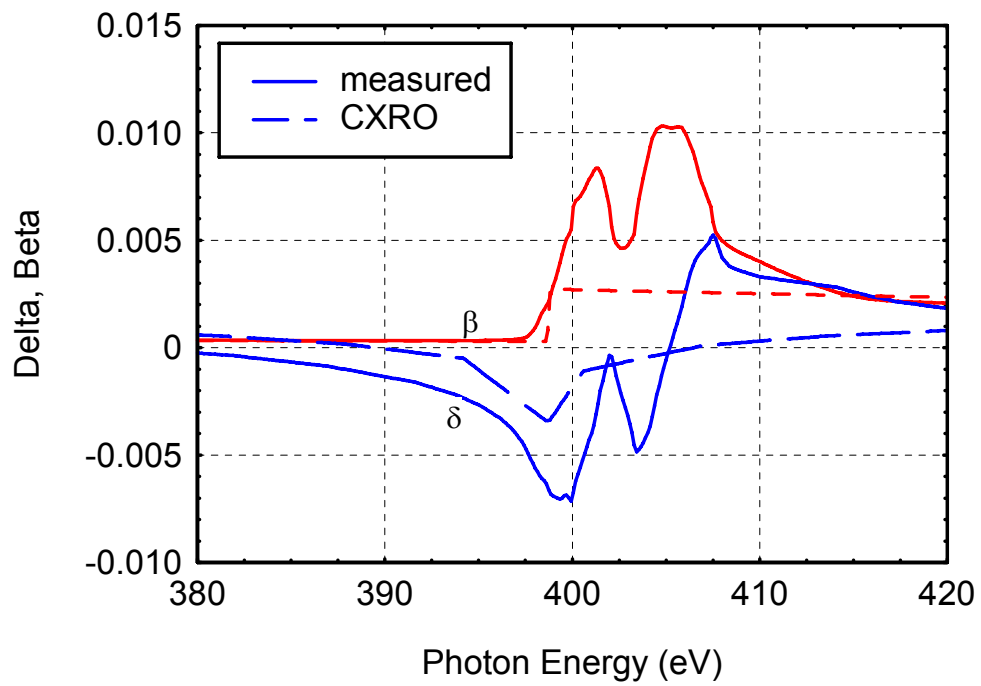

Figure 7. The optical constants near the Scandium $\mathrm{L}_{2,3}$ edge. Notice the drop in $\delta$ is lower than in the tabulated values which increases the theoretical reflectivity of Sc containing multilayer mirrors in this region. 


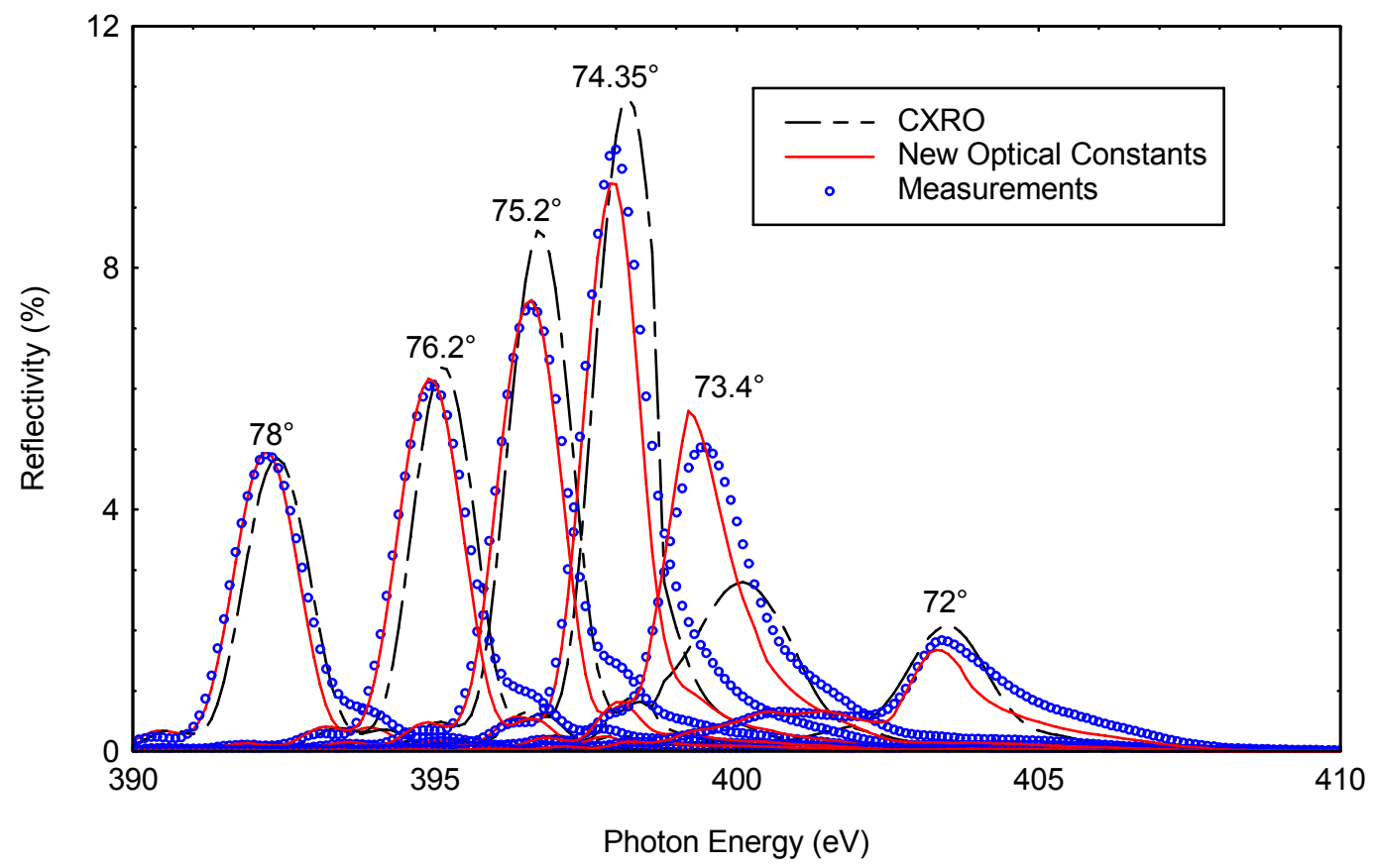

Figure 8. The reflectivity modeled with the old and new optical constants for a $\mathrm{Cr} / \mathrm{Sc}$ mirror are compared with measurements. The mirror parameters were $\mathrm{d}=1.6205 \mathrm{~nm}, \Gamma=.66$ and $\mathrm{N}=300$ bilayers. Note the improved agreement with the new optical constants especially near the edge. The highest reflectivity occurs at $398 \mathrm{eV}$ just below the $\mathrm{Sc} \mathrm{L}_{3}$ edge.

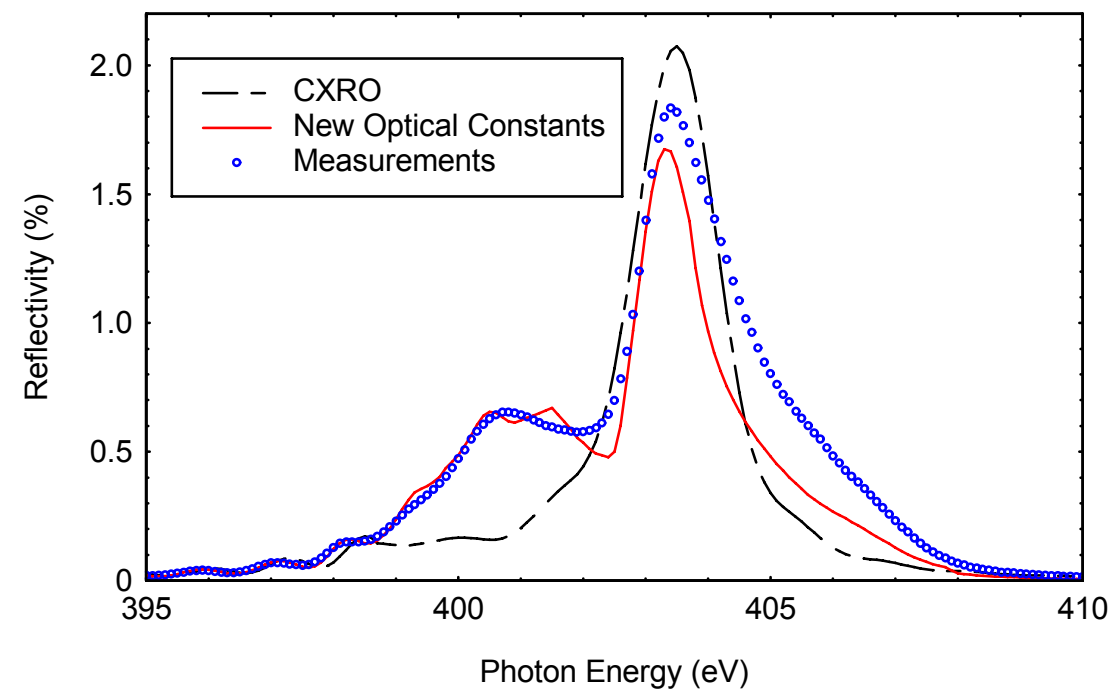

Figure 9. Reflectivity of the $\mathrm{Cr} / \mathrm{Sc}$ multilayer at 72 degrees where the Bragg peak occurs above the $\mathrm{Sc} \mathrm{L}$ edge. The model using the new optical constants better fits the side peak near $400 \mathrm{eV}$. 
Using the new index of refraction for Scandium a comparison to a $\mathrm{Cr} / \mathrm{Sc}$ multilayer was preformed. The $\mathrm{d}$ spacing of the multilayer was calculated using $\mathrm{Cu} \mathrm{K}_{\alpha}$ reflectometry. Gamma and the number of periods were obtained during the deposition of the mirror allowing us to model reflectivity using roughness as the only parameter. The multilayer was then scanned from 390 to $410 \mathrm{eV}$ (across the scandium edge) where its reflectivity would be the highest. Wavelength scans were preformed at .1 degrees of sample angle rotation. Thus the peak reflectivity should follow the Bragg law of $\lambda=2 \mathrm{~d} \sin (\theta)$, where $\theta$ is the grazing angle of incidence. The highest reflectivity was observed at $398 \mathrm{eV}$, just below the $\mathrm{Sc}_{3}$ edge. The old optical constants as well as the new optical constants were used to model the measured reflectivity curves. Both models used the same parameters $\left(d\right.$ and $\Gamma$ ) while the roughness was fitted for each using the $78^{\circ}$ data. Then the reflectivity was calculated at other angles up to and beyond the scandium edge (see graphs figures 8 and 9). Notice how well the model using the new optical constants tracks the data compared to previous constants. Even above the scandium edge the new model predicts the large tail of the data that is not predicted using the tabulated optical constants.

\section{CONCLUSION}

Precise measurements of the index of refraction of Scandium in the range of $50 \mathrm{eV}$ to $1,300 \mathrm{eV}$ are presented in this research. The experiment measured the absorption of Scandium and from these measurements allowed for the calculation of the optical constants. The results revealed a strong absorption structure around the Scandium $\mathrm{L}_{2,3}$ edge, that was not included in previous tabulations of Scandium absorption. The reflectivity modeled with the new optical constants was in good agreement with measurements on a $\mathrm{Cr} / \mathrm{Sc}$ multilayer mirror. The new optical constants will be available on the CXRO website at: http://www.cxro.lbl.gov/optical_constants/

\section{ACKNOWLEDGEMENTS}

The authors would like to thank Regina Soufli for helpful discussions in reviewing the data and Franklin Dollar for his help in obtaining the measurements. The work at the Berkeley Lab was supported by the U.S. Department of Energy, office of Basic Energy Sciences.

\section{REFERENCES}

\footnotetext{
${ }^{1}$ Fredrik Eriksson, Gran A. Johansson, Hans M. Hertz, Eric M. Gullikson, Ulrich Kreissig, and Jens Birch, " 14.5\% near-normal incidence reflectance of Cr Sc x-ray multilayer mirrors for the water window," Optics Letters, 28, No. 24, 2494 (Dec. 2003).

${ }^{2}$ B. L. Henke, E. M. Gullikson, and J. C. Davis, "X-ray interaction: photoabsorption, scattering, transmission, and reflection at $\mathrm{E}=50-30,000 \mathrm{eV}, \mathrm{Z}=1-92$,"At. Data Nucl. Data Tables 54, 181-343 (1993).

${ }^{3}$ E. M. Gullikson, S. Mrowka and B. B. Kaufmann "Recent developments in EUV reflectometry at the advanced light source” Proc. SPIE 4343 363-73 (2002)

${ }^{4}$ R.N.S. Sodhi and C.E. Brion, “ Reference Energies for Inner Shell Electron Energy-Loss Spectroscopy”, J. Elec. Spectr. and Rel. Phenom. 34, 363 (1984).

${ }^{5}$ E. M. Gullikson, "X-ray interactions with matter," http://www.cxro.lbl.gov/optical_constants/, currently available on the World Wide Web.

${ }^{6}$ Yu. A. Uspenskii, John F. Seely, N. L. Popov, A. V. Vinogradov, Yu. P. Pershin, and V. V. Kondratenko, "Efficient method for the determination of extreme-ultraviolet optical constants in reactive materials: application to scandium and titanium," Optical Society of America A-Optics \& Image Science, 21, No. 2 pp. 298 - 305 (2004).

7 J.H. Weaver, C. Krafka, D.W. Lynch, E.E. Koch, "Optical Properties of Metals", Physics Data 18-2 (1981).

${ }^{8}$ Regina Soufli, and Eric Gullikson, "Absolute photoabsorption measurements of molybdenum in the range $60-930 \mathrm{eV}$ for optical constant determination," Applied Optics 37, No. 10, 1713 (April 1998)
} 